\title{
Epidemiologic Change of Patients With Spinal Cord Injury
}

\author{
Ji Cheol Shin, $\mathrm{MD}^{1}$, Dae Hyun Kim, MD¹, Su Jin Yu, MD², Hea Eun Yang, MD', Seo Yeon Yoon, MD ${ }^{1}$ \\ ${ }^{1}$ Department of Rehabilitation and Research Institute of Rehabilitation, Yonsei University College of Medicine, Seoul; \\ ${ }^{2}$ Department of Rehabilitation Medicine, CHA Bundang Medical Center, CHA University, Bundang, Korea
}

\begin{abstract}
Objective To evaluate the epidemiologic change of patients with spinal cord injury who were admitted to a Rehabilitation Hospital, Yonsei University College of Medicine, during 1987-1996 and 2004-2008.

Methods Medical records of 629 patients with spinal cord injury admitted to the Rehabilitation Hospital, Yonsei University College of Medicine, from 2004 to 2008 were collected and reviewed retrospectively.

Results The male-to-female ratio decreased to 2.86:1, the mean age at injury increased, nontraumatic etiology increased, traffic accident remained to be the most common in traumatic spinal cord injury, and falling increased significantly. Tumor was the most common etiology in nontraumatic spinal cord injury, tetraplegia and incomplete injuries occurred more than paraplegia and complete injuries, indwelling catheter was the most common voiding method, and the duration of hospitalization decreased.

Conclusion Many trends changed in epidemiology of spinal cord injury.
\end{abstract}

Keywords Epidemiology, Spinal cord injuries

\section{INTRODUCTION}

There are many foreign studies regarding the epidemiology of patients with spinal cord injury (SCI). In the United States, the annual incidence of SCI is approximately 40 cases per million people in the general population. Thus, based on the United States population in

Received June 18, 2012; Accepted August 28, 2012

Corresponding author: Dae Hyun Kim

Department of Rehabilitation and Research Institute of Rehabilitation,

Yonsei University College of Medicine, 50 Yonsei-ro, Seodaemun-gu, Seoul 120-752, Korea

Tel: +82-2-2227-2681, Fax: +82-2-363-2795, E-mail: fly_boy_f@hotmail. com

(c) This is an open-access article distributed under the terms of the Creative Commons Attribution Non-Commercial License (http://creativecommons. org/licenses/by-nc/3.0) which permits unrestricted noncommercial use, distribution, and reproduction in any medium, provided the original work is properly cited.

Copyright $\odot 2013$ by Korean Academy of Rehabilitation Medicine
2010, approximately 12,400 new patients experienced SCI each year. Considering the population growth, this number will grow to 13,600 by 2020 , and 17,560 by 2050 [1]. A study in Norway reported that the annual incidence rate of SCI had grown from 6.2 per million in the period between 1952 and 1956 to 26.3 per million in the period between 1997 and 2001. The main reasons reported for the increase were car accidents and falls [2]. Other annual incidence rates of SCI that have been reported include 40.2 per million in Japan from 1990 to 1992, 18.0 per million in Jordan from 1988 to 1993, and 18.8 per million in Taiwan from 1992 to 1996 [3].

In the United States, the National Spinal Cord Injury Statistical Center (NSCISC) was established in the 1970s to continuously investigate the epidemiologic changes in SCI. It has reported changes, such as an increase in SCI prevalence among older people and females, and an increase in the percentage of patients with incomplete 

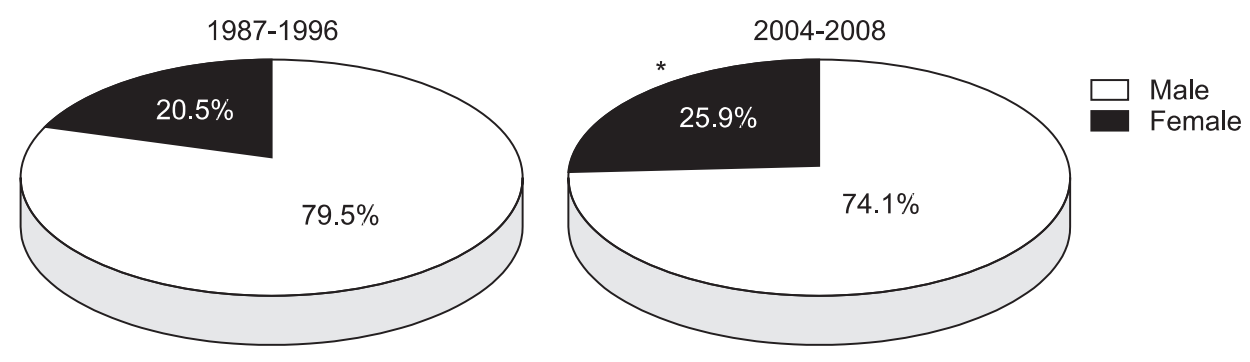

Fig. 1. Sex distribution changes between 1987-1996 and 2004-2008. ${ }^{*} \mathrm{p}<0.05$.

tetraplegia $[1,4,5]$.

In South Korea, no accurate statistical data exist on the prevalence and incidence of SCI, although it is expected that the epidemiologic changes will occur over time with an increase in the number of car accidents, developments in medicine, increase in the overall population, and population aging. Most epidemiologic research in Korea on patients with SCI consists of cross-sectional studies, and there is not enough research investigating the changes in epidemiologic factors. Hence, this study compared research [6] conducted on SCI patients who received hospital treatment from 1987 to 1996 with SCI patients who were hospitalized at the same institution from 2004 to 2008 to examine the changes in epidemiologic tendencies.

\section{MATERIALS AND METHODS}

\section{Study subjects}

The subjects selected were 629 patients with SCI, who were hospitalized and treated in the Department of Rehabilitation Medicine and Research Institute of Rehabilitation, Yonsei University College of Medicine, between March 1, 2004 and February 29, 2008.

\section{Methods}

This was a retrospective study. Patients' sex, age, age at the time of injury, cause of SCI, neurologic deficit, urination method, and period of hospitalization were investigated through medical records. Neurologic deficit was categorized according to the classifications used by the American Spinal Injury Association (ASIA) in its 2002 revised standards, and patient condition at hospitalization and at discharge were compared. Regarding the bladder evacuation techniques, urination through Crede's method, percussion, and Valsalva maneuver were categorized as reflex voiding. The changing trends were confirmed through comparison with a study [6] conducted on pa-

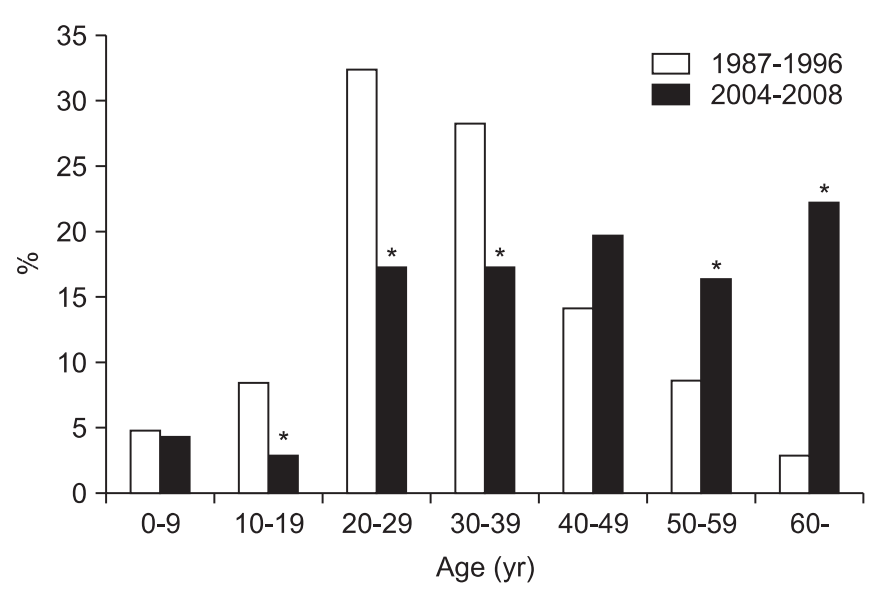

Fig. 2. Age at spinal cord injury changes between 19871996 and $2004-2008$. ${ }^{*} \mathrm{p}<0.05$.

tients hospitalized from 1987 to 1996, at the same institution. SPSS ver. 12 (SPSS Inc., Chicago, IL, USA) was used for statistical analysis, and the t-test, chi-square test, and Fisher's exact test were used.

\section{RESULTS}

\section{Change in sex and age}

Out of the total 629 patients, $466(74.1 \%)$ were male and $163(25.9 \%)$ were female, with a male-to-female ratio of approximately 2.86:1. From 1987 to 1996, there were a total of 590 patients, with 469 males and 121 females, and a male-to-female ratio of approximately 3.87:1. Comparing the two groups reveals a significant increase in the number of female patients with SCI (Fig. 1).

The mean age at the time of injury was 43.6 years, which had increased from the mean age of 32.3 years in the period from 1987 to 1996. Comparing the age groups between the two periods revealed a significant decrease in the number of patients aged between 10 and 39 years and a significant increase in those aged 50 or older (Fig. 2). 
Table 1. Etiology of spinal cord injury

\begin{tabular}{lcc}
\hline \multicolumn{1}{c}{ Etiology } & $\mathbf{1 9 8 7 - 1 9 9 6}$ & $\mathbf{2 0 0 4 - 2 0 0 8}$ \\
\hline Traumatic & $538(91.2)$ & $481(76.5)^{*}$ \\
Nontraumatic & $52(8.8)$ & $148(23.5)^{*}$ \\
\hline
\end{tabular}

Values are presented as number of cases (\%). ${ }^{*} \mathrm{p}<0.05$.

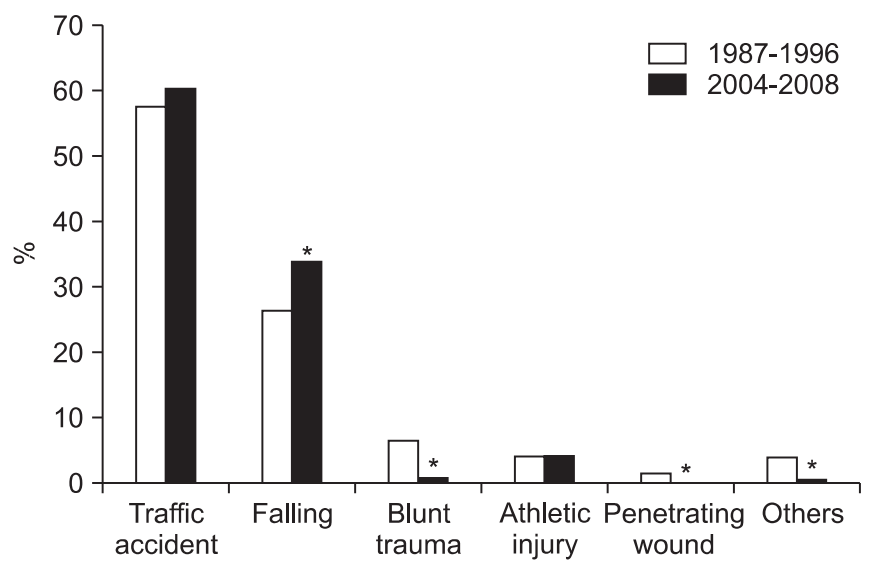

Fig. 3. Traumatic spinal cord injury changes between 1987-1996 and 2004-2008. ${ }^{*} \mathrm{p}<0.05$.

\section{Cause of spinal injury}

The cause of SCI was external trauma for $538(91.2 \%)$ out of 590 patients, from 1987 to 1996, and for 481 (76.5\%) out of 629 patients from 2004 to 2008 . The decreased ratio indicates that there were more injuries from nontraumatic causes in the later period (Table 1). Among traumatic causes, there was no change in the most common cause, car accidents, while injuries from falling showed a significant increase and blunt trauma and penetrating trauma showed a decreasing trend. There was no change in the ratio of sports injuries (Fig. 3). From 2004 to 2008, the most common cause of nontraumatic SCI was tumor, accounting for 50 (33.8\%) out of 148 patients. Other nontraumatic causes, in order of frequency, were spinal stenosis or disc rupture in $36(24.3 \%)$ patients, transverse myelitis in 23 (15.5\%) patients, arteriovenous malformation in $13(8.8 \%)$ patients, infection and spinal cord infarction in $9(6.1 \%)$ patients each, other epidural hemorrhage in $4(2.7 \%)$ patients, epidural abscess in 2 patients, and syringomyelia, which occurred in 2 patients after hydrocephalus and SCI after acupuncture, respectively. From 1987 to 1996, nontraumatic causes of SCI were, in order of frequency, spinal tuberculosis in $12(23.1 \%)$ out of 52 cases, disc rupture and transverse myelitis in 11 patients each $(21.2 \%)$, tumor in 10 patients $(19.2 \%)$, and
Table 2. Traumatic and nontraumatic spinal cord injury (SCI)

\begin{tabular}{lcc}
\hline & Traumatic SCI & Nontraumatic SCI \\
\hline Male & $379(78.8)$ & $88(59.5)$ \\
Age (yr) & $44.3 \pm 16.9$ & $46.6 \pm 20.3$ \\
DOH (day) & $55 \pm 39$ & $54 \pm 51$ \\
AIS & & \\
A & $247(51.4)$ & $18(12.2)^{*}$ \\
B & $73(15.2)$ & $10(6.8)^{*}$ \\
C & $87(18.1)$ & $45(30.4)^{*}$ \\
D & $74(15.4)$ & $75(50.7)^{*}$ \\
Total & 481 & 148 \\
\hline
\end{tabular}

Values are presented as number (\%) or mean \pm standard deviation.

DOH, duration of hospitalization; AIS, American Spinal Injury Association impairment scale.

${ }^{*} \mathrm{p}<0.05$.

arteriovenous malformation in 8 patients (15.4\%). Thus, the ratio of tumor and disc rupture increased, while spinal tuberculosis decreased.

\section{Comparison of external trauma and nontraumatic causes}

When the distribution of the ASIA impairment scale (AIS) was compared between SCI patients with traumatic and nontraumatic causes at hospitalization, there were more motor-complete injuries (AIS-A or B) for patients with traumatic SCI, while motor-incomplete injuries (AIS-C or D) were more common for patients with nontraumatic SCI (Table 2). There were no changes according to the traumatic and nontraumatic causes in the ratio of AIS-B, and C patients showing improvement in AIS at the time of discharge. Of the 481 patients with traumatic SCI, 379 were male and 103 were female, with a male-tofemale ratio of approximately $3.7: 1$. Of the 148 patients with nontraumatic SCI, 88 were male and 60 were female, with a male-to-female ratio of approximately 1.5:1. There was no statistically significant difference in the mean age of onset between traumatic (44.3 \pm 16.9$)$ and nontraumatic causes $(46.6 \pm 20.3)$. Patients in their 40 s were the largest age group among those with traumatic SCI, accounting for $101(21.0 \%)$ patients, followed in frequency by those in their 20s, with 95 (19.8\%) patients, and those in their 30 s, with $88(18.3 \%)$ patients. Patients in their 50s and 60 s were more common among those with nontraumatic SCI, with $26(17.6 \%)$ patients each. There was no statisti- 
cal difference in hospital stay between traumatic (55 \pm 39 days) and nontraumatic ( $54 \pm 51$ days) causes.

\section{Change in type of disability and severity of injury}

When categorizing the types of disabilities caused by SCI, there were more cases of tetraplegia (60.3\%) than paraplegia (39.7\%). Regarding severity of injury, there were more cases of incomplete paralysis $(58.8 \%)$ than complete paralysis (41.2\%). These results directly oppose those of 1987 to 1996 (Table 3). In the paraplegia group, there was a statistically significant increase in incomplete paralysis and a decrease in complete paralysis. Incomplete paralysis also increased in tetraplegia, but the change was not significant [4].

\section{AIS and neurologic recovery}

When patients were hospitalized in the Department of Rehabilitative Medicine, there was a relative decrease in AIS-A and increase in AIS-C and D, compared with the AIS from 1987 to 1996. The ratio that showed improvement in AIS at discharge compared with hospitalization

Table 3. Distribution of spinal cord injury by neurologic category

\begin{tabular}{lrc}
\hline & $\mathbf{1 9 8 7 - 1 9 9 6}$ & $\mathbf{2 0 0 4 - 2 0 0 8}$ \\
\hline Tetraplegia & & \\
Complete & $121(20.5)$ & $147(23.4)$ \\
Incomplete & $141(23.9)$ & $232(36.9)$ \\
Paraplegia & & \\
Complete & $229(38.8)$ & $112(17.8)^{*}$ \\
Incomplete & $99(16.8)$ & $138(21.9)^{*}$ \\
\hline
\end{tabular}

Values are presented as number (\%).

${ }^{*} \mathrm{p}<0.05$.

Table 4. Conversion rate of the American Spinal Injury Association impairment scale (AIS)

\begin{tabular}{|c|c|c|c|c|}
\hline \multirow[t]{2}{*}{ AIS } & \multicolumn{2}{|c|}{ Admission } & \multicolumn{2}{|c|}{$\begin{array}{c}\text { Conversion at } \\
\text { discharge }\end{array}$} \\
\hline & 1987-1996 & 2004-2008 & 1987-1996 & 2004-2008 \\
\hline A & $350(61.6)$ & $265(42.1)^{*}$ & $9(2.5)$ & $1(0.4)^{*}$ \\
\hline B & $97(17.1)$ & $83(13.2)$ & $12(12.4)$ & $2(2.4)^{*}$ \\
\hline $\mathrm{C}$ & $86(15.1)$ & $132(21.0)^{*}$ & $6(7.0)$ & $12(9.1)$ \\
\hline D & $35(6.2)$ & $149(23.7)^{*}$ & $0(0.0)$ & $0(0.0)$ \\
\hline Total & $568(100.0)$ & $629(100.0)$ & $36(6.3)$ & $15(2.4)$ \\
\hline
\end{tabular}

Values are presented as number (\%).

${ }^{*} \mathrm{p}<0.05$. significantly decreased in AIS-A and B, while no difference was seen in AIS-C (Table 4).

\section{Change in method of urination}

Reflex voiding was used by 481 (81.5\%) out of the total 590 patients from 1987 to 1996 . However, from 2004 to 2008, a urethral indwelling catheter was used by 243 (39\%) patients, clean intermittent catheterization by 191 (30.4\%) patients, and self-voiding by $153(24.3 \%)$ patients. Only 17 patients used reflex voiding (Table 5 ).

\section{Change in mean period of hospitalization}

The mean period of hospitalization of SCI patients continuously decreased over time throughout the periods from 1987 to 1991, 1992 to 1996, and 2004 to 2008. From 1987 to 1996 , patients with tetraplegia and complete damage had a relatively longer period of hospitalization, but from 2004 to 2008, there were no statistical differences between all the groups (Table 6).

Table 5. Change of voiding method

\begin{tabular}{lcc}
\hline \multicolumn{1}{c}{ Voiding method } & $\mathbf{1 9 8 7 - 1 9 9 6}$ & $\mathbf{2 0 0 4 - 2 0 0 8}$ \\
\hline Reflex voiding & $481(81.5)$ & $17(2.7)^{*}$ \\
$\begin{array}{l}\text { Clean intermittent } \\
\text { catheterization }\end{array}$ & $47(8.0)$ & $191(30.4)^{*}$ \\
\hline Self voiding & $36(6.1)$ & $153(24.3)^{*}$ \\
$\begin{array}{l}\text { External sphincterotomy } \\
\text { Urethral indwelling catheter }\end{array}$ & $11(1.9)$ & $0(0.0)^{*}$ \\
$\begin{array}{l}\text { Suprapubic indwelling } \\
\text { catheter }\end{array}$ & $6(1.0)$ & $245(39.0)^{*}$ \\
\hline Total & $590(100.0)$ & $629(100.0)$ \\
\hline
\end{tabular}

Values are presented as number (\%). ${ }^{*} \mathrm{p}<0.05$.

Table 6. Duration of hospitalization

\begin{tabular}{|cccc}
\hline & \multicolumn{3}{c}{ Hospital days } \\
\cline { 2 - 4 } & $\mathbf{1 9 8 7 - 1 9 9 1}$ & $\mathbf{1 9 9 2 - 1 9 9 6}$ & $\mathbf{2 0 0 4 - 2 0 0 8}$ \\
\hline Tetraplegia & & & \\
Complete & 198 & 116 & 56 \\
\hline Incomplete & 161 & 103 & 56 \\
\hline Paraplegia & & & \\
\hline Complete & 106 & 91 & 52 \\
\hline Incomplete & 84 & 92 & 51 \\
\hline
\end{tabular}




\section{DISCUSSION}

In Korea, $92 \%$ of patients with SCI were male in 1985 [7], but this ratio decreased to $78.6 \%$ from 1987 to 1996 [6]. In this research, the ratio decreased to $74.1 \%$, while the ratio of females increased to $25.9 \%$. In the United States, the female-to-male ratio showed an increasing trend from $18.2 \%$ in the 1970 s to $21.8 \%$ in 2000 [8]. This trend in the United States is thought to have arisen from the increase in the mean life expectancy, and the fact that the mean age at onset is higher in females than in males, rather than from the changing social role of women $[1,4]$. In this research, there were 103 female patients out of a total of 481 patients with traumatic SCI, which is a similar proportion to that in the United States [8]. Among nontraumatic SCI patients, there were 60 (40.5\%) female patients out of 148 total, which is a lower ratio than that of the $50 \%$ reported by New et al. [9] or McKinley et al. [10]. There was no difference in the mean age according to sex among both traumatic and nontraumatic causes. Among traumatic causes, car accidents were the most common, affecting 227 out of 378 male patients, and 63 out of 103 female patients; there was no difference between the sexes. These aspects of traumatic SCI are thought to result from the increased ratio of female patients due to increased participation of females in society and the workplace; this factor is different from the United States $[6,11]$. In the case of nontraumatic SCI, the ratio of female patients was lower than results reported in other countries, but there were limits to analyzing the changing trends, as there are insufficient past data regarding nontraumatic SCI in Korea.

In the United States, the mean age at SCI onset showed an increasing trend from 28.9 years in the 1970s to 38.0 years in 2000, and there was an increase in the age for all causes of SCI $[1,4,5]$. In Korea, too, the mean age at SCI onset increased from 29.6 years in the research of Nam et al. [7] reported in 1985 to 32.3 years in the research of Park et al. [6] in 1999 and 43.6 years in this research. Regarding patient age, there was a noticeable decrease in patients aged between 10 and 39 years, and an increase in those in their 50s and 60s. The ratio of SCI patients in their 60 s and older, especially, increased from $3.9 \%$ in the period from 1987 to $22.3 \%$ in 1996 , in this research. This increase is thought to be related to the increase in the mean age of the general population [4] and the increase in social activity of the aging population. Therefore, there is a need for treatment and management of elderly SCI patients.

SCI from traumatic causes decreased, while nontraumatic causes showed a significant increase. Among patients with traumatic SCI, car accidents were the most common cause, with $60.3 \%$. This is not significantly different from the ratio observed from 1987 to 1996 (57.6\%). In the United States, car accidents cause about half of traumatic SCI cases $[1,5]$, which is not significantly different from the ratio observed in the 1970s [1]. Among the traumatic causes, only falls showed a statistically significant increase compared with the past-being the most common cause of injury in elderly patients in their 60 s or older, especially while other causes showed no change or decrease. Falls were also the cause of injury in 58 (54.2\%) out of 107 patients in their 60s or older in this research. This fact is considered to be the result of increased mean life expectancy, and research in other countries has shown similar results $[1,5]$. Therefore, measures to prevent the elderly from falling down are urgently needed in our country. Causes of nontraumatic SCI, in order of frequency, were tumor, spinal stenosis or disc rupture, transverse myelitis, and arteriovenous malformation. As tumors grew more common as a cause of nontraumatic SCI, spinal tuberculosis displayed a decreasing trend. In the research of Citterio et al. [12] in 2004, tumors accounted for 81 out of 330 cases of nontraumatic SCI, showing similar tendencies to this study. It is thought that spinal tuberculosis decreased as a cause of SCI, due to the developments in the treatment for tuberculosis.

Regarding disability type and severity of injury, incomplete tetraplegia patients were the most common, accounting for $36.9 \%$, and the percentage of those with tetraplegia and incomplete paralysis showed a significant increase, compared with the results from 1987 to 1996 [6]. Exner and Meinecke [13] did not report any trends, but in the research of O'Connor [14] and Ahoniemi et al. [15], there was an increase in cervical injuries and incomplete injuries, similar to the results of this research. This trend is thought to arise from the development of prompt internal medicine and surgical treatment after SCI, which leads to an increase in incomplete paralysis [1], and from the fact that incomplete tetraplegia is the most typical injury type from falls of elderly patients [4]. As aging progresses in society, this tendency is expected to intensify, 
and considering that medical costs are higher for tetraplegic patients than for paraplegic patients [8], measures are needed to respond to the increasing economic burden.

When AIS at hospitalization and discharge were compared, the ratio of AIS-A and B patients showing improvement in AIS during hospitalization decreased in the 2004-2008 period, compared with the earlier period. One reason is thought to be the reduced period of hospitalization. There were limits to comparisons with precedent results, as only a small number of patients (1 patient, AIS-A; 2 patients, AIS-B) showed improvement. In subsequent studies, continuous monitoring of AIS improvement through outpatient clinics is needed.

Regarding method of urination, there was a significant decrease in patients using reflex voiding compared with 1987 to 1996. The proportions of patients using an indwelling catheter, clean intermittent catheterization, or self-voiding were similar, but all three methods showed a significant increase compared with the earlier period. In the past, using catheters was thought to cause urologic complications [6], but nowadays it is considered to be the optimal urination method, considering the surrounding environment of the patient, as well as the condition of the bladder. The above results are thought to reflect his changing trend.

The mean period of hospitalization was cut in half, compared with the past. In addition, in the past, tetraplegia and complete injury had longer periods of hospitalization. In this research, however, there were no differences in the number of days of hospitalization between tetraplegia and paraplegia, and between complete injury and incomplete injury. This is considered to reflect the influence of the current health insurance system in Korea, which sets limits according to the duration of hospitalization. Hence, it is necessary to perform effective rehabilitative treatment in a limited time frame through setting appropriate treatment goals and establishing treatment plans for individual patients at the early stages of hospitalization.

It is known that nontraumatic SCI patients are more likely to have incomplete injuries and fewer secondary complications compared with traumatic SCI patients [16]. This research also showed that nontraumatic SCI patients included fewer AIS-A and B patients, but more C and D patients. When the AIS at hospitalization and discharge were compared in nontraumatic SCI patients, 1 out of 10 AIS-B patients and 5 out of 45 AIS-C patients showed improvement. Due to the small number of patients, there were limitations to comparing the improvements in AIS at hospitalization and discharge of traumatic and nontraumatic SCI patients.

This research is limited in that it was performed on patients of a single institution. This method is appropriate, however, to observe epidemiologic changes in SCI patients in Korea, as it compared research results performed at the same institution in different time periods.

In conclusion, the SCI patients, who were hospitalized in the Department of Rehabilitation Medicine and Research Institute of Rehabilitation, Yonsei University College of Medicine between 2004 and 2008, showed the following epidemiologic changes compared with patients who were hospitalized between 1987 and 1996. 1) There was a significant increase in the percentage of female SCI patients, as the male-to-female ratio changed from 3.87:1 to 2.86:1. 2) Age at the time of injury increased from the mean of 32.3 to 43.6 years. SCI rates decreased among those aged 10 to 39 years, and increased among those in their 50s or older. 3) The cause of injury is showing a tendency of decreasing traumatic SCI and increasing nontraumatic SCI. For traumatic SCI, car accidents were still the cause of the majority of cases, but falls as the cause of injury showed a significant increase. For nontraumatic SCI, tumors were the most common cause with $33.8 \%$, followed in frequency by spinal stenosis, disc rupture, transverse myelitis, and arteriovenous malformation. 4) Patients with motor-complete injuries were more common among traumatic SCI than nontraumatic SCI, while patients with motor-incomplete injuries were more common among the nontraumatic SCI than traumatic SCI. 5) Regarding neurological damage categorization, there were more cases of tetraplegia, with $60.3 \%$, than paraplegia, and more cases of incomplete paralysis, with 58.8\%, than complete paralysis. These results contrasted with results from the past. 6) The mean duration of hospitalization decreased by approximately 50 days, and there were no differences in the duration of hospitalization, according to the type of injury. And 7) In order of frequency, urination methods most performed were indwelling catheter, clean intermittent catheterization, and self-voiding, while reflex voiding showed a significant decrease. 


\section{CONFLICT OF INTEREST}

No potential conflict of interest relevant to this article was reported.

\section{REFERENCES}

1. Devivo MJ. Epidemiology of traumatic spinal cord injury: trends and future implications. Spinal Cord 2012;50:365-72.

2. Hagen EM, Eide GE, Rekand T, Gilhus NE, Gronning M. A 50-year follow-up of the incidence of traumatic spinal cord injuries in Western Norway. Spinal Cord 2010;48:313-8.

3. Wyndaele M, Wyndaele JJ. Incidence, prevalence and epidemiology of spinal cord injury: what learns a worldwide literature survey? Spinal Cord 2006;44:5239.

4. Jackson AB, Dijkers M, Devivo MJ, Poczatek RB. A demographic profile of new traumatic spinal cord injuries: change and stability over 30 years. Arch Phys Med Rehabil 2004;85:1740-8.

5. DeVivo MJ, Chen Y. Trends in new injuries, prevalent cases, and aging with spinal cord injury. Arch Phys Med Rehabil 2011;92:332-8.

6. Park CI, Shin JC, Kim SW, Jang SH, Chung WT, Kim HJ. Epidemiologic study of spinal cord injury. J Korean Acad Rehabil Med 1999;23:267-75.

7. Nam YH, Juhn YS, Sung IY, Kang SK, Oh CH. Current status on 110 members in the Korean Welfare Association of spinal cord disabled. J Korean Acad Rehabil Med 1985;9:120-5.

8. Ho CH, Wuermser LA, Priebe MM, Chiodo AE, Scelza
WM, Kirshblum SC. Spinal cord injury medicine. 1 . Epidemiology and classification. Arch Phys Med Rehabil 2007;88(3 Suppl 1):S49-54.

9. New PW, Simmonds F, Stevermuer T. A populationbased study comparing traumatic spinal cord injury and non-traumatic spinal cord injury using a national rehabilitation database. Spinal Cord 2011;49:397-403.

10. McKinley WO, Seel RT, Hardman JT. Nontraumatic spinal cord injury: incidence, epidemiology, and functional outcome. Arch Phys Med Rehabil 1999;80:61923.

11. Kuhn W, Zach GA, Kochlin P, Urwyler A. Comparison of spinal cord injuries in females and in males, 19791981 Basle. Paraplegia 1983;21:154-60.

12. Citterio A, Franceschini M, Spizzichino L, Reggio A, Rossi B, Stampacchia G, et al. Nontraumatic spinal cord injury: an Italian survey. Arch Phys Med Rehabil 2004;85:1483-7.

13. Exner G, Meinecke FW. Trends in the treatment of patients with spinal cord lesions seen within a period of 20 years in German centers. Spinal Cord 1997;35:4159.

14. O'Connor PJ. Forecasting of spinal cord injury annual case numbers in Australia. Arch Phys Med Rehabil 2005;86:48-51.

15. Ahoniemi E, Alaranta H, Hokkinen EM, Valtonen K, Kautiainen H. Incidence of traumatic spinal cord injuries in Finland over a 30-year period. Spinal Cord 2008;46:781-4.

16. Chapman J. Comparing medical complications from nontraumatic and traumatic spinal cord injury [abstract]. Arch Phys Med Rehabil 2000;81:1264. 